\title{
Modelling of Nef interaction with ABCA1 revealed potential binding sites for inhibitor compounds
}

\author{
Anastasia A. Anashkina \\ EIMB RAS, Moscow, Russia \\ nastya@eimb.ru
}

\author{
Yaroslav V. Tkachev \\ EIMB RAS, Moscow, Russia \\ yat@eimb.ru
}

Alexei A. Adzhubei

EIMB RAS, Moscow, Russia alexei.adzhubei@eimb.ru

\begin{abstract}
The effect of HIV-1 Nef protein on the demyelination of central nervous system cells is mediated by its effect on the cholesterol transporter protein ABCA1. To determine the possible interactions of Nef-ABCA1, an expert model of the cytoplasmic fragment $\mathrm{ABCA1}$ was constructed and modelling of the reciprocal binding sites in the Nef and ABCA1 structures was carried out. This made it possible to determine the interface for the interaction of proteins and localize binding sites in them.
\end{abstract}

Keywords - Nef, ABCA1, demyelination, model, binding site

Motivation and aim

\section{Motivation}

It is known that the Nef inhibits the activity of ABCA1, disrupts its maturation in the endoplasmic reticulum, and also induces the internalization and degradation of the membrane protein ABCA1. Chaperon calnexin, with which Nef forms a complex, plays an important role in this process.

Aim

Earlier we had shown that Nef interacts with the structural epitope ABCA1, which consists of sites in the two adjacent cytoplasmic domains [1]. While the site in the C-terminal domain ABCA1 was identified by us earlier, other possible interaction sites in ABCA1, as well as sites in Nef responsible for the interaction, remain unknown. This information is of major importance for the rational design of inhibitors.

\section{Methods}

To perform the task of searching for other binding sites of the ABCA1 cytoplasmic domain with Nef, an expert model of the cytoplasmic plus transmembrane domains of ABCA1, equilibrated by molecular dynamics, was built. Next, modeling of binding sites in the Nef and ABCA1 structures was performed using global docking, and employing our previously developed approach and server [2].

The structure of ABCA1 cytoplasmic domains D1 (8471324) and D2 (1870-2261) was modeled by the servers Phyre2, iTasser, RaptorX, M4T, SwissModel. In addition, a model of the full-sized protein ABCA1 was built using the SwissModel server, based on the incomplete PDB 5XJY structure (with multiple missing fragments) obtained by electron microscopy. Further construction of the model structure was carried out by expert modeling.

For subsequent equilibration by molecular dynamics, a model was prepared, which contained the transmembrane and cytoplasmic parts of ABCA1 and a fragment of the lipid membrane. The system was minimized in 5,000 steps, then balanced in 1 fs steps for 75,000 steps and 2 fs steps for 150,000 steps using temperature and pressure coupling. For a 60 ns trajectory, the NPT ensemble was modeled at a temperature during the $310 \mathrm{~K}$ simulation. All simulations were performed using the full atomic force field CHARMM36 and GROMACS software.

The previously constructed model of the structure of Nef protein [3] was also equilibrated by molecular dynamics method. Given the mobility of the Nef protein loops, 5 different conformation clusters were selected that most fully represent the space of conformational states. Representative structures of these clusters were used for docking.

The model of the cytoplasmic part of ABCA1, obtained after molecular dynamics, was used for docking with models of the Nef structure. Docking was carried out between the Nef structure models and the structure model of the cytoplasmic part of ABCA1 without a membrane, using ZDock, SwarmDock, GrammX and ClusPro servers. A total of 80 docking models of the interaction of cytoplasmic part of ABCA1 with Nef were obtained.

After excluding docking models in which Nef overlapped the structural region occupied by the membrane, eighteen ABCA1 - Nef models were selected.

\section{Results}

Considering that the N-terminus of Nef protein is lipidated at the Gly2 residue with the myristic acid residue, which serves to anchor Nef in the membrane, there remains only one ABCA1-Nef docking model, satisfying such spatial criteria. Using the QASDOM server [2], we determined the ABCA1 and Nef protein regions that form the interaction interface. The binding sites are centered on residues for which the largest number of intermolecular atomic interactions is shown. For ABCA1, these are Ser1023, Leu1026, Leu1972, Thr1261, Pro1267, Leu1282, Pro1284, Thr1305. For Nef: Asp32, Asp40, Asn51, Cys59, Trp61, Tyr85, Asp127. These putative binding sites will be used for structurally oriented virtual screening.

\section{ACKNOWLEDGMENT}

This work was supported by a grant from RFBR 17-5430021 NIZ_a.

\section{REFERENCES}

[1] Jacob D. et al. The ABCA1 domain responsible for interaction with HIV-1 Nef is conformational and not linear // Biochem Biophys Res Commun 2014. Vol. 444. P. 19.

[2] Anashkina AA. et al. Meta-server for automatic analysis, scoring and ranking of docking models // Bioinformatics 2018. Vol. 34. P. 297.

[3] Hunegnaw R. et al. Interaction Between HIV-1 Nef and CalnexinHighlights // Arteriosclerosis, thrombosis, and vascular biology 2016. Vol. 36. P. 1758. 